\title{
Estimativa da evapotranspiração de referência com base em modelos de temperatura do ar e radiação solar para o Município de Estreito - MA
}

\author{
Reference evapotranspiration estimate based on air temperature and solar radiation models for the
} Municipality of Estreito - MA

Estimación de evapotranspiración de referencia basada en modelos de temperatura del aire y radiación solar para el Municipio de Estreito - MA

Elton Ferreira Lima

ORCID: https://orcid.org/0000-0002-5725-6811 Universidade Federal de Santa Maria, Brasil E-mail: elton110@hotmail.com

Jossimara Ferreira Damascena ORCID: https://orcid.org/0000-0002-7020-8507 Universidade Federal do Tocantins, Brasil E-mail: jossi_agro@hotmail.com Juliana Andrade Pereira ORCID: https://orcid.org/0000-0003-4131-0489
Universidade Estadual da Região Tocantina do Maranhão, Brasil E-mail: juliana_andradep@hotmail.com

Fernando Almeida da Conceição ORCID: https://orcid.org/0000-0001-8653-9995 Universidade Estadual da Região Tocantina do Maranhão, Brasil E-mail: feernando86@gmail.com

Pedro Luid De Sousa Oliveira

ORCID: https://orcid.org/0000-0003-3317-6590 Universidade Estadual da Região Tocantina do Maranhão, Brasil E-mail: pluid93@hotmail.com

Potiara Oliveira Diniz

ORCID: https://orcid.org/0000-0003-4391-290X Universidade Federal Rural do Semi-Árido, Brasil E-mail: potiara_diniz@hotmail.com

Wendel dos Santos Moraes

ORCID: https://orcid.org/0000-0002-6791-4478 Universidade Federal do Tocantins, Brasil E-mail: wendel.moraes@mail.uft.edu.br Cristiane Matos da Silva

ORCID: https://orcid.org/0000-0002-6416-4413 Universidade Estadual da Região Tocantina do Maranhão, Brasil E-mail: cristiane.silva@uemasul.edu.br

\begin{abstract}
Resumo
A escassez de recursos hídricos é o fator mais limitante da produção agrícola no município de Estreito, MA. A irregularidade na distribuição das chuvas durante o ano, característica das regiões tropicais, faz com que a atividade agrícola em Estreito, assim como em outras cidades do estado do Maranhão reduzam na época de seca. Diante desse cenário, a busca por meios que visem melhorar a eficiência da irrigação para o gerenciamento sustentável da água se torna indispensável. O presente estudo teve como objetivo realizar um estudo comparativo dos métodos de estimativa da evapotranspiração de referência baseados na temperatura do ar e radiação solar, com o método padrão de PenmanMonteith, no munícipio de Estreito - MA. Os dados meteorológicos necessários para a execução desse trabalho foram adquiridos do banco de dados do Instituto Nacional de Meteorologia, utilizando-se uma série histórica contínua de onze anos dos elementos climáticos que foram utilizados nas equações que compõem os diferentes métodos. Foram calculadas as estimativas da evapotranspiração de referência para Penman-Monteith-FAO e os demais métodos. Para a avaliação do desempenho estatístico dos modelos, foram empregados os indicadores do coeficiente de correlação (r), coeficiente de determinação $\left(\mathrm{R}^{2}\right)$, índice de concordância (d) e o índice de desempenho (c). Os modelos da Radiação FAO 24, Turc e Makkink apresentaram melhores desempenho quando comparados ao método padrão de Penman Monteith, podendo servir como métodos alternativos para estimativa da evapotranspiração de referência no município de Estreito - MA.
\end{abstract}

Palavras-chave: Recursos hídricos; Escassez; Produção agrícola. 


\begin{abstract}
The scarcity of water resources is the most limiting factor of agricultural production in the municipality of Estreito, MA. The irregularity in the distribution of rainfall during the year, which is characteristic of tropical regions, means that agricultural activity in Estreito, as well as in other cities in the state of Maranhão, reduces during the dry season. In view of this scenario, the search for means that aim to improve the efficiency of irrigation for the sustainable management of water becomes indispensable. The present study aimed to carry out a comparative study of the reference evapotranspiration estimation methods based on air temperature and solar radiation, with the standard Penman-Monteith method, in the municipality of Estreito - MA. The meteorological data necessary for the execution of this work were acquired from the database of the National Institute of Meteorology, using an eleven-year continuous historical series of the climatic elements that were used in the equations that make up the different methods. The reference evapotranspiration estimates for Penman-Monteith-FAO and the other methods were calculated. For the evaluation of the statistical performance of the models, the indicators of the correlation coefficient (r), determination coefficient $\left(\mathrm{R}^{2}\right)$, agreement index $(\mathrm{d})$ and the performance index (c) were used. The FAO 24, Turc and Makkink Radiation models showed better performance when compared to the standard Penman - Monteith method, and can serve as alternative methods for estimating reference evapotranspiration in the municipality of Estreito - MA.
\end{abstract}

Keywords: Water resources; Scarcity; Agricultural production.

\title{
Resumen
}

La escasez de recursos hídricos es el factor más limitante de la producción agrícola en el municipio de Estreito, MA. La irregularidad en la distribución de las lluvias durante el año, característica de las regiones tropicales, hace que la actividad agrícola en Estreito, así como en otras ciudades del estado de Maranhão, se reduzca durante la estación seca. Ante este escenario, la búsqueda de formas de mejorar la eficiencia del riego para la gestión sostenible del agua se vuelve indispensable. El presente estudio tuvo como objetivo realizar un estudio comparativo de los métodos de estimación de evapotranspiración de referencia basados en la temperatura del aire y la radiación solar, con el método estándar Penman-Monteith, en el municipio de Estreito - MA. Los datos meteorológicos necesarios para la ejecución de este trabajo fueron adquiridos de la base de datos del Instituto Nacional de Meteorología, utilizando una serie histórica continua de once años de los elementos climáticos que se utilizaron en las ecuaciones que componen los diferentes métodos. Se calcularon las estimaciones de evapotranspiración de referencia para Penman-Monteith-FAO y los otros métodos. Para la evaluación del desempeño estadístico de los modelos se utilizaron los indicadores del coeficiente de correlación (r), coeficiente de determinación $\left(\mathrm{R}^{2}\right)$, índice de concordancia $(\mathrm{d})$ e índice de desempeño (c). Los modelos Radiación FAO 24, Turc y Makkink mostraron un mejor desempeño en comparación con el método estándar de Penman-Monteith y pueden servir como métodos alternativos para estimar la evapotranspiración de referencia en el municipio de Estreito-MA.

Palabras clave: Recursos hídricos; Escasez; Producción agrícola.

\section{Introdução}

A escassez de recursos hídricos é o fator mais limitante a produção agrícola no município de Estreito, MA. Em muitos casos, a baixa produtividade é atribuída ao baixo nível tecnológico empregado na agricultura bem como a sistemas de gestão agrícola ineficientes (Lima et al., 2020). Embora isso seja verdade, a insuficiência de água é de longe o fator mais limitante com chuvas, que segundo o INMET, variam de 357,20 mm.ano-1 a 1.618,6 mm.ano-1 e distribuídas irregularmente em duas estações (seca e chuvosa).

A irregularidade na distribuição das chuvas durante o ano, característica das regiões tropicais, faz com que a atividade agrícola em Estreito, assim como em outras cidades do estado do Maranhão reduzam na época da seca. Como consequência dessa redução, ocorre uma elevação no preço dos produtos e importações de outras regiões, visto que aproximadamente $100 \%$ das terras do estado usadas para produção de alimento é baseada na agricultura de sequeiro (Lima et al., 2020). Nesse contexto, a irrigação se constitui em uma alternativa para minimizar os efeitos da seca e garantir a segurança alimentar da população nessa região.

Em contraste a crescente dependência da produção irrigada, a disponibilidade de água para irrigação vem sofrendo uma drástica redução em virtude da combinação de períodos de estiagem e a competição que engloba usuários para fins agrícolas, individuais e industriais (Hallal et al., 2017). Diante desse cenário, a busca por meios que visem melhorar a eficiência da irrigação para o gerenciamento sustentável da água se torna indispensável (Júnior et al., 2013). Para uma melhor 
eficiência dos sistemas de irrigação, a estimativa precisa da evapotranspiração de referência (ETo) é fundamental.

A evapotranspiração é uma das variáveis mais importantes do ciclo da água no contínuo solo-planta-atmosfera e pode ser determinada por uma grande variedade de métodos que na maioria das vezes são complicados, demorados e caros (Raziei \& Pereira, 2013; Carvalho et al., 2015). Entre os vários métodos existentes, a Organização das Nações Unidas para Agricultura e Alimentação (FAO) adotou o método de Penman-Monteith como o único método padrão para estimativa da ETo (Djaman, 2015; Pacheco et al., 2014).

Entretanto, devido à grande quantidade de insumos meteorológicos necessários para sua execução, o uso desse método tem ficado restrito em várias regiões, principalmente nos países em desenvolvimento onde o acesso a esses dados ainda é limitado (Alencar et al., 2011). Nesse contexto, pesquisas que busquem a previsão da evapotranspiração sob condição de dados limitados são inovadoras, uma vez que, um método para ser considerado ideal deve requerer o mínimo possível de variáveis de entrada sem afetar a precisão do modelo (Silva, Campos \& Azevedo, 2009; Ribeiro, Simeão \& Santos, 2016).

O município de estreito contém uma única estação meteorológica automática e essa ainda apresenta algumas limitações espaciais. Além disso, nenhum trabalho com o objetivo de comparar apenas modelos baseados na temperatura do ar ou radiação solar para estimar a evapotranspiração de referência no município foi encontrado, evidenciando assim a necessidade deste estudo. Pensando nisso, o objetivo desse trabalho foi comparar o desempenho de oito modelos baseados apenas na temperatura do ar e radiação, na tentativa de encontrar um método mais simples de estimativa da ETo e que apresente boa precisão em relação ao método padrão que é complexo e de difícil aplicação prática.

\section{Metodologia}

\subsection{Caracterização geográfica e climática da região de estudo}

Localizado no Nordeste brasileiro, especificamente no estado do Maranhão, o município de Estreito está localizado

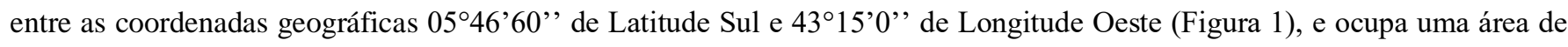
aproximadamente $2.719 \mathrm{~km}^{2}$. A área de estudo possui abundantes recursos de luz e calor, mas a distribuição das chuvas é bastante irregular tornando a atividade agrícola insustentável. 
Figura 1. Localização do Município de Estreito.

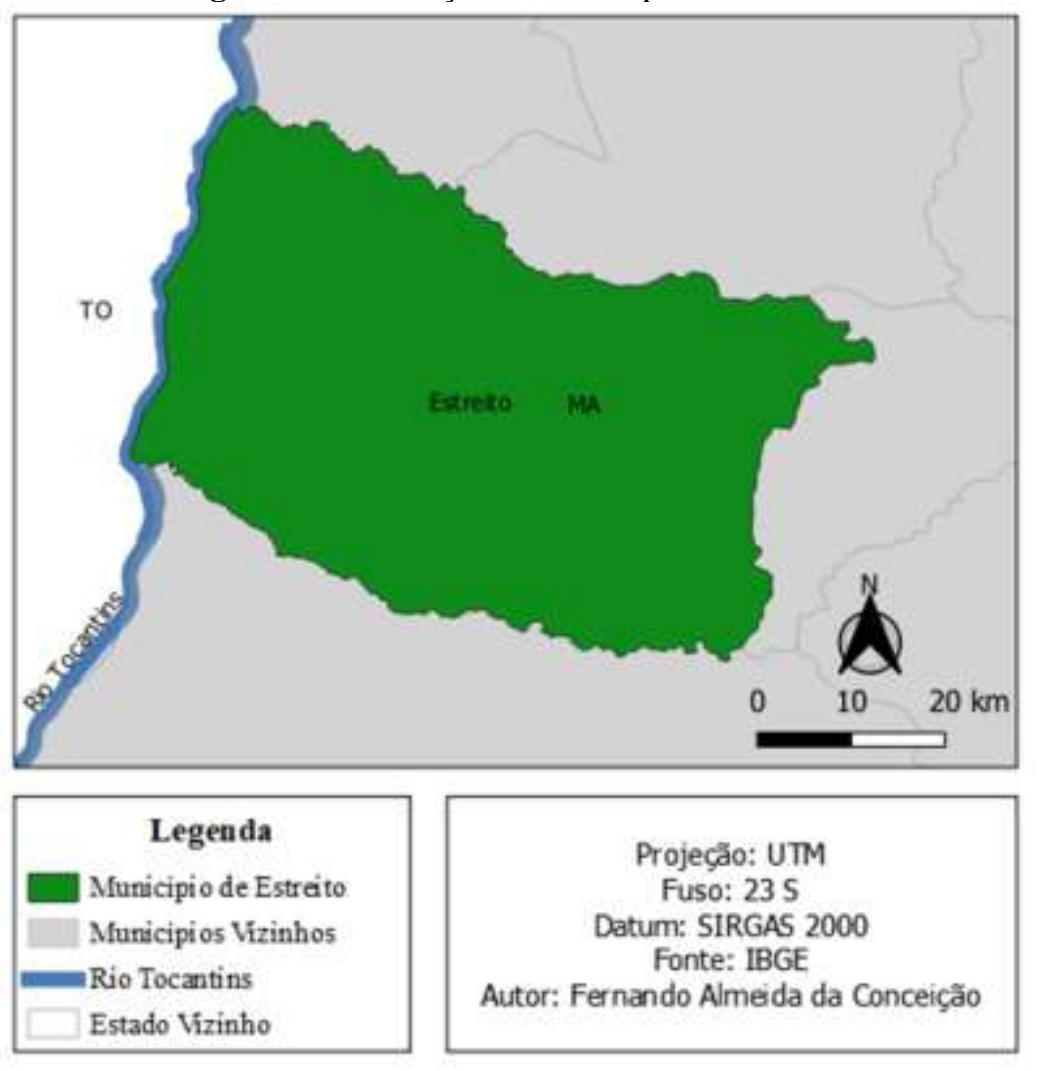

Fonte: Autores (2021).

Dados meteorológicos diários, incluindo temperatura máxima (Tmax) e mínima (Tmin), radiação solar (Rs), umidade relativa (UR), precipitação $(\mathrm{P})$ e velocidade do vento $\left(\mathrm{U}_{2}\right)$ a 2 metro de altura foram obtidos de uma estação meteorológica automática, pertencentes a rede de estações do Instituto Nacional de Meteorologia (INMET) e localizada no município. A qualidade dos dados foi rigorosamente controlada conforme a metodologia adotada por Sales et al. (2018), além disso, os dias com ausência de dados foram eliminados. Uma caracterização climática da estação estudada incluindo o período analisado após verificação dos dados é apresentada na Tabela 1.

Tabela 1. Caracterização da estação de coleta dos dados e das condições climáticas de Estreito, MA no período de 28/02/2008 à 30/06/2019. Tar: temperatura média do ar; UR: umidade relativa média do ar; $\mathrm{U}_{2}$ : velocidade do vento a $2 \mathrm{~m}$ de altura; $\mathrm{P}$ : precipitação média ao longo dos 11 anos de estudo.

\begin{tabular}{ccccccccc}
\hline Estação & $\begin{array}{c}\text { Código } \\
\text { MMO }\end{array}$ & $\begin{array}{c}\text { Latitude } \\
\mathbf{S}\end{array}$ & $\begin{array}{c}\text { Longitude } \\
\mathbf{W}\end{array}$ & $\begin{array}{c}\text { Altitude } \\
(\mathbf{m})\end{array}$ & $\begin{array}{c}\text { Tar } \\
\left({ }^{\circ} \mathbf{C}\right)\end{array}$ & $\begin{array}{c}\text { UR } \\
(\boldsymbol{\%})\end{array}$ & $\begin{array}{c}\mathbf{U}_{2} \\
\left(\mathbf{m . s}^{\mathbf{1}}\right)\end{array}$ & $\begin{array}{c}\mathbf{P} \\
(\mathbf{m m} / \mathbf{a n o})\end{array}$ \\
\hline Estreito & 81863 & $06^{\circ} 39$ & $47^{\circ} 25^{\prime}$ & 180 & 26,24 & 75,07 & 1,17 & 968,00 \\
\hline
\end{tabular}

Fonte: Autores (2021).

\subsection{Estimativa da evapotranspiração}

A pesquisa é de campo, de natureza qualitativa, pois foi realizada a comparação entre modelos buscando um mais simples e que represente bem os valores reais de evapotranspiração local

Para o cálculo da evapotranspiração de referência foram selecionados quatro modelos baseados na temperatura do ar e quatro baseados na radiação solar (Tabela 2). Esses modelos foram escolhidos por necessitarem apenas de dados de temperatura ou radiação, ou ambos, como parâmetro de entrada para estimar a evapotranspiração. 
Tabela 2. Métodos de estimativa da evapotranspiração de referência e suas equações.

\begin{tabular}{|c|c|c|}
\hline Método & Abreviação & Equação \\
\hline \multicolumn{3}{|c|}{ Modelos baseados na temperatura do ar } \\
\hline Budyko (1974) & BK & ETo $=0,2 \mathrm{~T}$ \\
\hline Camargo (1971) & $\mathrm{CM}$ & $\mathrm{ETo}=0,012 \cdot \mathrm{R}_{\mathrm{a} \text { mm dia }}-1 \times \mathrm{T}$ \\
\hline Hagreaves e Samani (1985) & HS & ETo $=0,408 \cdot 0,0023 \cdot(\mathrm{T}+17,8) \cdot\left(\mathrm{T}_{\max }-\mathrm{T}_{\min }\right)^{0,5} \cdot \mathrm{R}_{\text {amm.dia }^{-1}}$ \\
\hline \multirow{2}{*}{ Linacre (1977) } & \multirow[t]{2}{*}{$\mathrm{LN}$} & \multirow{2}{*}{ ETo $=\frac{500\left(\frac{\mathrm{T}+0,006 \mathrm{xh}}{100-\varphi}\right)+15\left(\mathrm{~T}-\mathrm{T}_{\mathrm{po}}\right)}{(80-\mathrm{T})}$} \\
\hline & & \\
\hline \multicolumn{3}{|c|}{ Modelos baseados na radiação solar } \\
\hline Makkink (1957) & $\mathrm{Mk}$ & 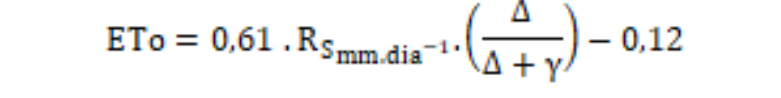 \\
\hline Jensen Heise (1963) & $\mathrm{JH}$ & $\mathrm{ETo}=\mathrm{R}_{\mathrm{S}_{\mathrm{mm} \text { dia }}}{ }^{-1}(0,025 . \mathrm{T}+0,078)$ \\
\hline Turc (1955) & $\mathrm{TC}$ & $\mathrm{ETo}=0,013 \cdot\left[T_{\max }(T \max +15)^{-1} \times(50+23,88 R n)\right.$ \\
\hline Radiação FAO 24 & RA & ETo $=-0,3+$ cl. W. $_{2} \frac{\mathrm{Rs}}{2,45}$ \\
\hline
\end{tabular}

Legenda: ETo = evapotranspiração de referência $\left(\mathrm{mm} \mathrm{dia}^{-1}\right) ; \Delta=$ declividade da curva de pressão $\left(\mathrm{kPa}^{\circ} \mathrm{C}^{-1}\right) ; \mathrm{Rn}=$ saldo de radiação $\left(\mathrm{MJ}^{-2}\right.$ dia $\left.^{-1}\right) ; \mathrm{G}=$ fluxo de calor $\left(\mathrm{MJ} \mathrm{m}^{-2} \mathrm{dia}^{-1}\right) ; \gamma=$ constante psicrométrica $\left(\mathrm{MJ} \mathrm{kg}^{-1}\right) ; \mathrm{T}=$ temperatura média $\left({ }^{\circ} \mathrm{C}\right)$; U2 = velocidade do vento $(\mathrm{m} \mathrm{s}$ $\left.{ }^{1}\right)$; es = pressão de saturação de vapor $(\mathrm{kPa})$; ea = pressão de vapor $(\mathrm{kPa})$; Tmax $=$ temperatura máxima $\left({ }^{\circ} \mathrm{C}\right)$; Tmin $=$ temperatura mínima $\left({ }^{\circ} \mathrm{C}\right) ; \operatorname{Ramm} \cdot \mathrm{dia}^{-1}=$ Radiação extraterrestre $\left(\mathrm{mm} \cdot \mathrm{dia}^{-1}\right) ; \mathrm{Rsmm} \cdot \mathrm{dia}^{-1}=$ radiação global $\left(\mathrm{mm} \cdot \mathrm{dia}^{-1}\right) ; \lambda=$ calor latente de vaporização $\left(\mathrm{MJ} \cdot \mathrm{mm}{ }^{-1}\right)$; $\mathrm{URm}=$ umidada relativa média $(\%) ; \mathrm{h}=$ altitude do local $(\mathrm{m}) ; \varphi=$ latitude em módulo (graus); Tpo = temperatura do ponto de orvalho $\left({ }^{\circ} \mathrm{C}\right)$. Fonte: Autores (2021).

A metodologia utilizada como base comparativa para o processo de avaliação dos métodos supracitados foi a de Penman-Monteith (PM), parametrizado pelo Boletim FAO 56 como o único método padrão para estimativa da ETo (Allen et al., 1998), sendo calculado pela seguinte expressão:

$$
\text { ETo }=\frac{\left[0,408 \cdot \Delta \cdot(R n-G)+\left(\frac{900 \cdot U_{2}}{T+273}\right) \cdot(\text { es }- \text { ea })\right]}{\Delta+\gamma \cdot\left(1+0,34 \cdot U_{2 j}\right.}
$$

Em que: ETo = evapotranspiração de referência $\left(\mathrm{mm}\right.$ dia-1); $\Delta=$ declividade da curva de pressão $\left(\mathrm{kPa}{ }^{\circ} \mathrm{C}-1\right) ; \mathrm{Rn}=$ saldo de radiação (MJ m-2 dia-1); $\mathrm{G}=$ fluxo de calor $(\mathrm{MJ} \mathrm{m}-2$ dia-1); $\gamma$ = constante psicrométrica $(\mathrm{MJ}$ kg-1); $\mathrm{T}=$ temperatura média $\left({ }^{\circ} \mathrm{C}\right) ; \mathrm{U} 2$ = velocidade do vento $(\mathrm{m} \mathrm{s}-1)$; es = pressão de saturação de vapor $(\mathrm{kPa})$; ea = pressão de vapor $(\mathrm{kPa})$.

\subsection{Avaliação de desempenho}

O desempenho das diferentes metodologias de estimativa da ETo em relação ao método de Penman Monteith foram calculados por meio de planilha eletrônica e avaliados utilizando a seguinte estatística: índice de concordância de Willmott (d), coeficiente de correlação de Pearson (r), coeficiente de determinação $\left(R^{2}\right)$ resultante da regressão linear, e índice de confiança/desempenho (c) proposto por Camargo \& Sentelhas (1997), equações 2, 3, e 4 respectivamente.

$$
\mathbf{d}=1-\left[\frac{\left[\Sigma(\mathrm{Pi}-\mathbf{0 i})^{2}\right]}{\sum(|\mathrm{Pi}-\mathbf{0}|+|\mathbf{0 i}+\mathbf{0}|)^{2}}\right]
$$




$$
\begin{aligned}
& \mathbf{r}=\frac{\sum_{\mathrm{i}=1}^{\mathrm{N}}(\mathrm{Oi}-\mathbf{0}) \cdot(\mathrm{Pi}-\mathrm{P})}{\sqrt{\sum_{\mathrm{i}=1}^{\mathrm{N}}(\mathrm{Oi}-\mathbf{O})^{2}} \cdot \sqrt{\sum_{\mathrm{i}=1}^{\mathrm{N}}(\mathrm{Pi}-\mathrm{P})^{2}}} \\
& \mathbf{c}=\mathbf{r} \cdot \mathbf{d}
\end{aligned}
$$

Em que: $\mathrm{Pi}$ = evapotranspiração estimada pelo método testado (mm.d-1); P a média da evapotranspiração do método testado $\left(\mathrm{mm} \cdot \mathrm{d}^{-1}\right)$; Oi = evapotranspiração estimada pelo método padrão $\left(\mathrm{mm} \cdot \mathrm{d}^{-1}\right) ; \mathrm{O}=$ média dos valores observados pelo método padrão (mm.d-1) e n é o número de dias compreendido no período.

Os valores do índice "c", proposto por Camargo e Sentelhas (1997), variam de 0 para nenhuma concordância, a 1 para concordância perfeita entre os dados, podendo seus resultados serem qualificados de acordo com a Tabela 3.

Tabela 3. Critério de interpretação do desempenho pelo índice "c".

\begin{tabular}{ll}
\hline c & Desempenho \\
\hline$>\mathbf{0 , 8 5}$ & Ótimo \\
$\mathbf{0 , 7 6}$ a 0,85 & Muito Bom \\
$\mathbf{0 , 6 6}$ a 0,75 & Bom \\
$\mathbf{0 , 6 1}$ a 0,65 & Mediano \\
$\mathbf{0 , 5 1}$ a 0,60 & Sofrível \\
$\mathbf{0 , 4 1}$ a 0,50 & Mau \\
$\leq \mathbf{0 , 4 0}$ & Péssimo \\
\hline
\end{tabular}

Fonte: Camargo e Sentelhas (1997).

\section{Resultados e Discussão}

Estão apresentados na Figura 2 os gráficos e o modelo resultante da regressão linear entre a ETo estimada pelos métodos baseados na temperatura do ar e o método de Penman-Monteith (PM). Os resultados mostraram um baixo ajuste para todos os modelos, isso pode ser observado com base no coeficiente de determinação que variou de 0,26 (Camargo) a 0,63 (Linacre). Esses baixos valores de $\mathrm{R}^{2}$ indicam uma grande variância dos resíduos, portanto, uma péssima explicação do comportamento dos dados nesses modelos.

A análise da Figura 3 também mostrou que com exceção do modelo de Hagreaves e Samani, todas as demais metodologias baseadas na temperatura do ar apresentaram tendência de superestimar a ETo observada pelo método de Penman-Monteith. Esse fato pode ser observado analisando a distribuição dos valores da regressão em relação a linha 1:1, onde percebe-se que para as metodologias de Linacre, Camargo e Budiko grande parte dos pontos ficaram acima dessa linha indicando uma superestimativa. 
Figura 2. Relação entre a $E T_{o}$ diária estimada pelo método de Penman-Monteith (ETo-PM) e os métodos baseados na temperatura do ar, para a região de Estreito - MA. A linha tracejada representa a relação 1:1.
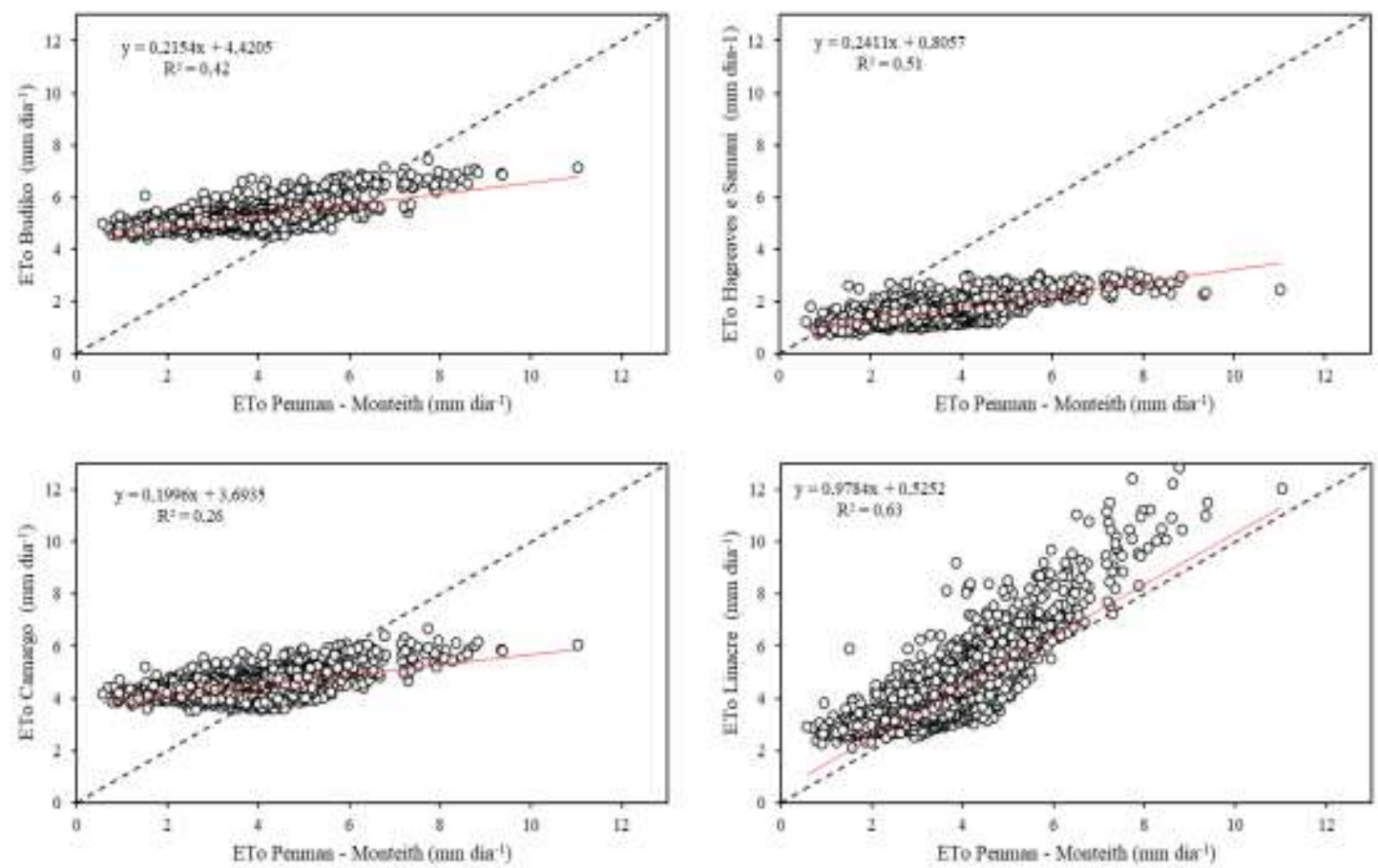

Fonte: Autores (2021).

Esses resultados podem ser explicados porque, apesar da facilidade de medida e de ser usada em diversos modelos agrícolas, sobretudo, no uso de métodos para cálculo da ETo e da evapotranspiração das culturas, a temperatura do ar é uma variável bastante influenciada pela mudança climática e portanto, modelos que utilizam apenas essa variável como dado de entrada para o cálculo da ETo, podem não se ajustar ao método padrão de Penman-Monteith (Santana et al., 2018).

Somado a isso, o fato de que os modelos/métodos aqui testados foram desenvolvidos para condições climáticas diferentes das de onde esse trabalho foi realizado, esse é um fator bem interessante a ser observado, uma vez que, o desempenho das abordagens baseadas na temperatura em um determinado local pode envolver flutuações importantes e ser fortemente dependente do contexto climático local específico, onde a temperatura pode não ser suficiente para uma explicação adequada da variabilidade ETo. Além disso, por não levar em consideração o efeito da radiação solar, déficit de pressão de vapor ou duração da insolação, que desempenham um papel importante para explicar ETo em regiões úmidas e locais com altas taxas de precipitação, onde a flutuação de ET pode estar geralmente mais ligado a variações desses fatores do que a variações de temperatura.

Corroborando os resultados aqui encontrados, vários outros estudos encontrados na literatura apontam tendências de superestimativas da ETo quando os modelos de temperatura são empregados no cálculo dessa variável. Santana et al. (2018) avaliando métodos de estimativa da ETo no município de Balsas, MA, encontraram tendências de superestimativas da ETo para as metodologias de Linacre, Camargo e Hagreaves e Samani. Gurski et al. (2016), comparando diferentes metodologias para aferir a ETo em Curitiba/PA, observaram aptidão de superestimativa da ETo com a metodologia de Linacre.

Para os modelos baseados na radiação solar, a Figura 3 mostra os gráficos de dispersão resultantes da regressão linear desses modelos em relação ao método de Penman-Monteith. A análise da Figura 3 permite inferir que esses modelos apresentaram um melhor ajuste ao método de PM quando comparado aos modelos baseados na temperatura (Figura 2). O coeficiente de determinação para esses modelos variou de 0,70 (Makkink) a 0,85 (Radiação FAO24) indicando que os valores 
da ETo calculada por esses métodos foram bem próximos daqueles obtidos pelo método de Penman-Monteith.

Figura 3. Relação entre a $\mathrm{ET}_{\mathrm{o}}$ diária estimada pelo método de Penman-Monteith $\left(\mathrm{ET}_{\mathrm{0}}-\mathrm{PM}\right)$ e os métodos baseados na radiação solar, para a região de Estreito - MA. A linha tracejada representa a relação 1:1.
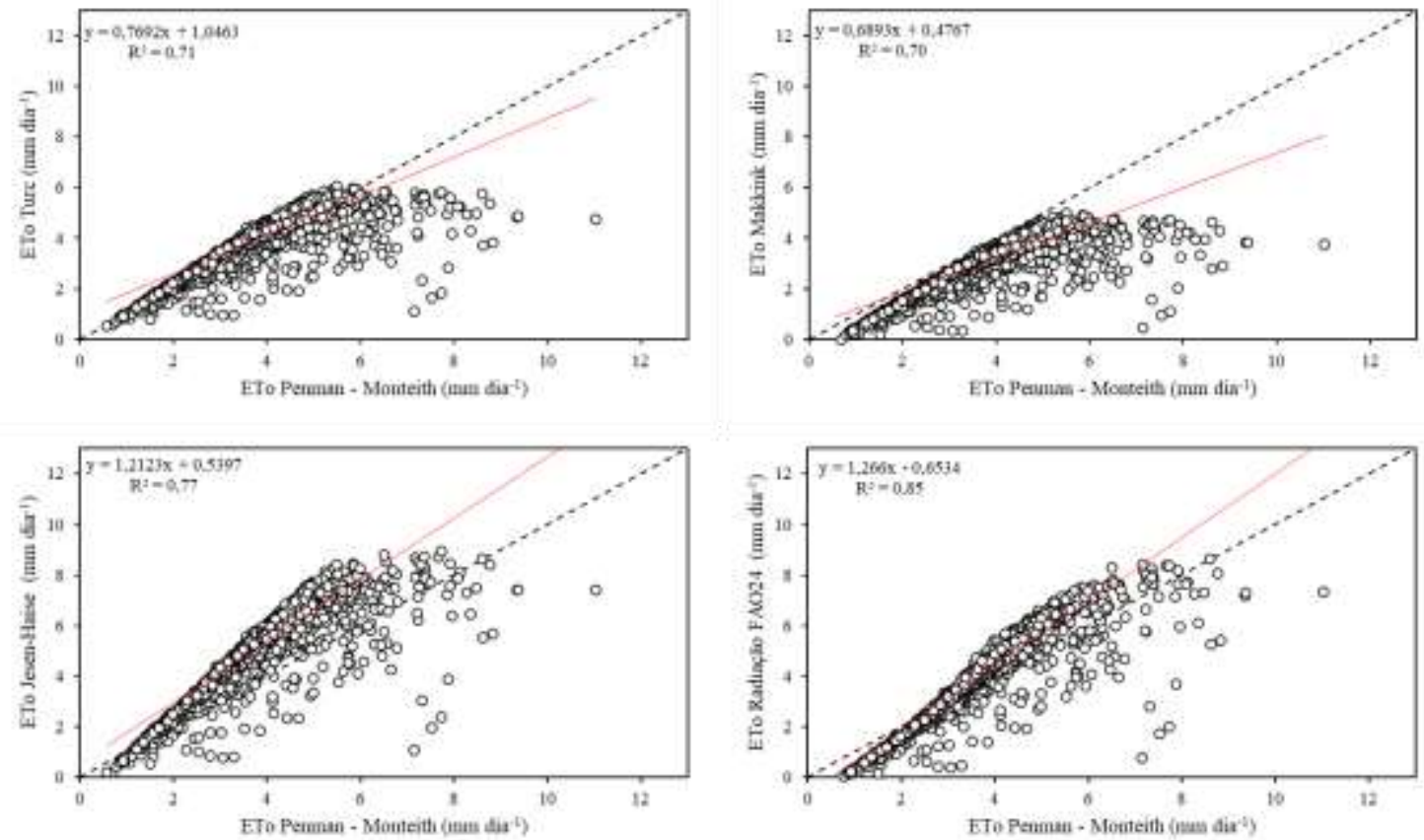

Fonte: Autores (2021).

Semelhante aos métodos baseados na temperatura, os modelos baseados na radiação também apresentaram tendências de superestimar a ETo, obtida pelo método de Penman-Monteith, a exceção é o método de Makkink que ao contrário dos demais apresentou tendência de subestimar os valores de ETo obtidos pelo método de PM. Essa tendência de subestimativa da ETo pelo método de Makkink está associada ao fato de que este foi desenvolvido para regiões frias (Rosemberg, Blade \& Verma, 1983) apresentando baixos valores de ETo quando empregado em regiões quentes, para superar esse fator é interessante fazer a calibração do modelo para a região aqui estudada.

Outros trabalhos com métodos de estimativa da evapotranspiração desenvolvidos em cidades do Maranhão também encontraram tendências de subestimativas da ETo por esse modelo, como no estudo de Santana et al. $(2018$, 2019) em Balsas. Os resultados aqui encontrados também estão de acordo aos encontrados por Vescove e Turco (2005) os quais comparando três metodologias de estimativa da evapotranspiração na região de Araraquara/SP, também verificaram que o método de Makkink subestimou a ETo em relação à Penman-Monteith (EToPM), nos dois períodos analisados.

As superestimativas observadas pelos métodos de Turc, Jensen-Heise e Radiação FAO 24, assim como a subestimativa da ETo obtida com o método de Makkink podem estar associadas ao fato de que esses modelos foram desenvolvidos para condições climáticas diferentes das de onde o presente estudo foi desenvolvido, sendo necessário um ajuste nos parâmetros de suas equações para obtenção de valores de ETo mais significativos. Entretanto, apesar de terem superestimado os valores de ETo obtidos pelo método de Penman-Monteith, no geral, esses métodos apresentaram bons resultados.

Pilau et al. (2012), corroborando com esses resultados, testando diferentes metodologias de estimativa da ETo nas localidades de Frederico Westphalen e Palmeira das Missões, RS, concluíram que o método da Radiação FAO 24, que incluem dados de radiação solar incidente como variável de entrada, pode substituir o método de Penman-Monteith (FAO), porém tem 
tendência de subestimar a ETo.

Turco, Faria e Fernandes (2005), verificaram superestimativas da ETo com o método de Jensen-Heise no município de Mutoca - PE. Por outro lado, diferente dos resultados aqui encontrados, Rojas e Sheffield (2013), em estudo no nordeste de Louisiana, EUA, constataram que o modelo de Turc subestimou a ETo padronizada em $10 \%$.

Embora os valores para o coeficiente de determinação tenham sido relativamente baixos para os modelos de temperatura e altos para os modelos de radiação, a adoção apenas do $\mathrm{R}^{2}$ como indicador da qualidade de um método, torna-se inadequada, visto que o $\mathrm{R}^{2}$ não estabelece a grandeza das discrepâncias entre um valor determinado e um estimado (Júnior et al., 2013; Barros et al., 2009). Assim, a análise de desempenho conjunta de um método, deve ser feita com base no índice de concordância (d), que fornece o grau de exatidão entre os parâmetros analisados, ou seja, relaciona a diferença entre os valores estimados pelos diferentes métodos e os valores observados pelo método de PM; o coeficiente de correlação (r), que mensura o grau de associação entre os modelos em análise fornecendo uma indicação de precisão do modelo; e o índice "c", que indica o desempenho do método, através do produto entre os índices r e d (Camargo \& Sentelhas, 1997). Os valores para esses coeficientes estão apresentados na Tabela 4.

Tabela 4. Desempenho dos métodos de estimativa da ETo baseados na temperatura do ar e radiação solar, com base no índice de Wilmott (d), coeficiente de correlação de Pearson (r) e índice de desempenho proposto por Camargo e Sentelhas (c"). BK: Budyko; HS: Hagreaves e Samani; CM: Camargo; LN: Linacre; TC: Turc; JH: Jensen Heise; MK: Makkink; RA: Radiação FAO 24.

\begin{tabular}{lcccc}
\hline \multicolumn{5}{c}{ Métodos baseados na temperatura do ar } \\
\hline Índice & ETo (BK) & ETo (HS) & ETo (CM) & ETo (LN) \\
\hline $\mathbf{d}$ & 0,50 & 0,41 & 0,56 & 0,84 \\
$\mathbf{r}$ & 0,64 & 0,72 & 0,80 & 0,51 \\
c" $^{\prime \prime}$ & 0,32 & 0,29 & 0,45 & 0,43 \\
Desempenho & Péssimo & Mediano & Mediano & Péssimo \\
\hline \multicolumn{5}{c}{ Métodos baseados na radiação solar } \\
\hline Índice & ETo (TC) & ETo (JH) & ETo (MK) & ETo (RA) \\
\hline d & 0,91 & 0,70 & 0,79 & 0,91 \\
r & 0,84 & 0,88 & 0,84 & 0,92 \\
c" & 0,76 & 0,61 & 0,66 & 0,84 \\
Desempenho & Muito bom & Mediano & Bom & Muito bom \\
\hline \multicolumn{5}{c}{ Fonte: Autores (2021). }
\end{tabular}

Para os métodos baseados na temperatura os valores do índice de concordância variaram de 0,41 (Hagreaves e Samani) a 0,84 (Linacre). Evidentemente, entre os modelos baseados na temperatura, o modelo de Linacre foi o que melhor se ajustou ao método de Penman-Monteith com base nesse coeficiente. Entretanto, esse mesmo comportamento não foi observado para o coeficiente de correlação e índice de confiança o que resultou em um desempenho classificado como Péssimo.

O péssimo desempenho encontrado pelo método de Linacre pode ser explicado porque além da temperatura esse método utiliza apenas a temperatura do ar e do ponto de orvalho e a altitude como variável do local, limitando a representatividade das condições climáticas para efeito de estimativa da ETo. Diferente dos resultados aqui encontrados, Jung et al. (2016) e Santana et al. (2019) encontraram desempenho sofrível utilizando a metodologia de Linacre para estimativa da ETo.

Para os métodos de Camargo e Hargreaves-Samani foram observadas correlações altas, com "r" de 0,80 e 0,72, respectivamente, entretanto esse comportamento não foi observado para o índice de concordância e índice de confiança o que resultou em um desempenho Mediano. Os resultados aqui encontrados podem ser explicados de acordo com Sentelhas et al. 
(2001), porque, originalmente, o método de Hargreaves-Samani foi desenvolvido para clima semiárido, e se baseia apenas em dados de temperatura, fornecendo em muitos locais valores diferentes dos encontrados pelo método padrão, o que aqui pode ser facilmente observado com base no índice de concordância que foi bem baixo $(d=0,41)$ indicando um alto desvio entre os valores de ETo obtidos por esse método em relação aos valores obtidos pelo método de Penman-Monteith.

Resultados diferentes aos aqui encontrados foram obtidos por Ribeiro, Simeão e Santos (2016) e por Pereira et al. (2009) que em seus trabalhos encontraram desempenho mediano e sofrível para esse método no período considerado seco. O método de Camargo foi o que apresentou o índice de confiança mais alto (dentro todos os métodos avaliados) e resultou em um desempenho Mediano. Porém, Cunha, Magalhães e Castros (2013), explica que o método de Camargo é derivado do método de Thornthwaite, e funciona eficazmente para regiões de clima tropical e equatorial úmidos, diferente do clima da região onde o presente estudo foi realizado.

Ainda com base na tabela 4 pode se observar, que o método de Budyko apresentou um índice de confiança de 0,32 resultando em um desempenho Péssimo. Fato este que pode ser comprovado nas Figura 2, onde esse método apresentou pontos com grandes dispersões ao redor da linha de tendência, indicando que não é um bom método para estimar a evapotranspiração no local de estudo. O que está de acordo com Araújo et al. (2010) onde o método de Budyko foi encontrado como o método que mais superestimou a ETo.

Para os métodos baseados na radiação solar os valores do índice de concordância variaram de 0,70 (Jensen-Haise) a 0,91 (Radiação FAO 24 e Turc). Dessa forma, os modelos da Radiação FAO 24, Turc e Makkink foram os que melhor se ajustaram ao método de Penman-Monteith com base nesse coeficiente. O mesmo comportamento foi observado para o índice de confiança o que resultou nos desempenhos Muito bom e Bom, respectivamente. Júnior et al. (2012), encontraram resultados semelhantes na região de Aquidauana/MS, testando o comportamento de metodologias de estimativa da ETo diária, entre os anos de 2008 a 2010, observaram bom desempenho com a metodologia de Turc.

Tagliaferre et al. (2012) encontraram desempenho Muito Bom utilizando a metodologia da Radiação FAO 24 para estimativa da ETo. No que se refere a metodologia de Jensen-Haise, embora este tenha apresentado uma correlação muito alta, observa-se que esse método apresentou um índice de confiança de 0,61 resultando em um desempenho Mediano, não sendo recomendado para substituição ao método de Penman-Monteith.

\section{Conclusão}

Os modelos da Radiação FAO 24, Turc e Makkink apresentaram melhores desempenho quando comparados ao método padrão de Penman - Monteith, podendo servir como métodos alternativos para estimativa da evapotranspiração de referência no município de Estreito - MA.

Mais estudos são necessários com os demais métodos aqui testados, pois apesar da facilidade de aplicação para o cálculo da ETo não se ajustaram ao método padrão. $\mathrm{O}$ uso de tais métodos poderia levar a erros na lâmina de água a ser aplicada as culturas. Estudos futuros focados na calibração local desses métodos é uma alternativa interessante a ser testada.

\section{Referências}

Alencar, L. P., Sediyama, G. C., Wanderley, H. S., Almeida, T. S., \& Delgado, R. C. (2011). Avaliação de métodos de estimativa da evapotranspiração de referência para três localidades no norte de Minas Gerais. Revista Engenharia na Agricultura, 19, 437449.

Allen, R. G., Pereira, L. S., Raes, D., \& Smith, M. (1998). Crop evapotranspiration: Guidelinesfor Computing Crop Water Requirements. Irrigation and Drainage Paper. FAO, 300(9), D05109.

Araújo, E. M., Oliveira, J. B., Araújo, E. M., Ledo, E. R. F., \& Silva, M. G. (2010). Desempenho de métodos de estimativa de ETo correlacionados com a equação padrão Penman-Monteith Fao-56, em cidades do estado do Ceará. Acta Tecnológica, 5(2), 84-101. 
Barros, V. R., Souza, A. P., Fonseca, D. C., \& Silva, L. B. D. (2009). Avaliação da evapotranspiração de referência na Região de Seropédica, Rio de Janeiro, utilizando lisímetro de pesagem e modelos matemáticos. Revista Brasileira de Ciências Agrárias, 4(2), 198-203.

Camargo, A. P., \& Sentelhas, P. C. (1997). Avaliação do desempenho de diferentes métodos de estimativa da evapotranspiração poten cial no Estado de São Paulo, Brasil. Rev. Bras. Agrometeorol, 5(1), 89-97.

Carvalho, D. F., Rocha, H. S., Bonomo, R, \& Souza, A. P. (2015). Estimativa da evapotranspiração de referência a partir de dados meteorológicos limitados. Pesquisa Agropecuária Brasileira, 50, 111.

Cunha, F. F., Magalhães, F. F., \& Castros, M. A. (2013). Métodos para estimativa da evapotranspiração de referência para Chapadão do Sul-MS. Engenharia na Agricultura, 21(2).

Djaman, K. (2015). Evaluation of sixteen reference evapotranspiration methods under sahelian conditions in the Senegal River Valley. Journal of Hydrology: Regional Studies, 3, 139-159.

Gurski, B. C., Souza, J. L. M., Jerszurki, D., Schäfer, R. F., \& Schäfer, H. (2016). Métodos alternativos de estimativa da evapotranspiração de referência anual e nas diferentes estações do ano em Curitiba-PR, Brasil. Revista Cultura Agronômica, 25(2), 155-166.

Hallal, M. O. C., Schöffel, E. R., Brixner, G. F. \& Cunha A. R. (2017). Métodos de estimativa da evapotranspiração de referência para Pelotas, Rio Grande do Sul. Rev. Cienc. Agrar, 60(1), 1-10.

Jung, L. H., Biscaro, G. A., Oliveira, G. Q., Alves, M. A., \& Giacon, G. M. (2017). Estimativa da evapotranspiração de referência em uma região do Alto Pantanal. MAGISTRA, 28(2), 168-177.

Júnior, E. D. F., Lopes, A. S., Oliveira, G. Q., \& Jung, L. H. (2012). Métodos empíricos para estimativa da evapotranspiração de referência para Aquidauana, MS. Irriga, 17(4), $418-434$.

Lima, E. F., Moraes, R. G. S., Sousa K. S. C., Fonseca, B.L.A.S., Damascena, J. F., Lima, M. A. S., et al (2020). Estimativa da evapotranspiração de referência (ETo) diária em Balsas/MA baseada apenas na temperatura do ar. Competência técnica e responsabilidade social e ambiental nas ciências agrárias 3.22, $22-8$.

Pacheco, A. P., Cerqueira, M. A., Silva, B. B., \& Nicácio, R. M. (2014). Análise espaço temporal da evapotranspiração na área de preservação ambiental da ilha de Santa Rita, Alagoas, Brasil. Revista Árvore, 38(3), 453-460.

Pereira, D. D. R., Yanagi, S. D. N. M., Mello, C. R. D., Silva, A. M. D., \& Silva, L. A. D. (2009). Desempenho de métodos de estimativa da evapotranspiração de referência para a região da Serra da Mantiqueira, MG. Ciência Rural, 39(9), 2488-2493.

Pilau, F. G., Battisti, R., Somavilla, L., \& Righi, E. Z. (2012). Desempenho de métodos de estimativa da evapotranspiração de referência nas localidades de Frederico Westphalen e Palmeira das Missões, RS. Ciência Rural, 42(2), 283-290.

Raziei, T., \& Pereira, L. S. (2013). Estimation of ETo with Hargreaves Samani and FAO PM temperature methods for a wide range of climates in Iran. Agricultural Water Management, 121, 118 .

Ribeiro, A. A., Simeão, M., \& Santos, A. R. B. (2016). Comparação de métodos de estimativa da evapotranspiração de referência no período chuvoso e seco em Piripiri (PI). Revista Agrogeoambiental, 8(3), 89-100.

Rojas, J. P., \& Sheffield, R. E. (2013). Evaluation of daily reference evapotranspiration methods as compared with the ASCE-EWRI Penman-Monteith equation using limited weather data in northeast Louisiana. Journal of Irrigation and Drainage Engineering, 139, $285-292$.

Rosemberg, N. J., Blade, B. L., \& Verma, S. B. (1983). Microclimate: the biological environment. (2a ed.), John Wiley \& Sons, 495.

Sales, R. A., Oliveira, E. C., Lima, M. J. A., Gelcer, E. M., Santos, R. A., \& Lima, C. F. (2018). Ajuste dos coeficientes das equações de estimativa da evapotranspiração de referência para São Mateus, ES. Irriga, 23(1), 154-167.

Santana, J. S., Lima, E. F., Damascena, J. F., \& Fernandes, M. C. (2019). Desempenho de equações agrometeorológicas para as estações seca e chuvosa para a cidade de Sorriso/MT. Enciclopédia biosfera, 16(29), 623.

Santana, J. S., Lima, E. F., Silva, W. A., Fernandes, M. C., \& Ribeiro, M. I. D. (2018). Equações de estimativa da evapotranspiração de referência (ETo) para a região de Balsas-MA. Enciclopédia biosfera, 15(27), 1.

Sentelhas, P. C. (2001). Agrometeorologia aplicada à irrigação. In: Miranda JH, Pires RCM. Manejo de Irrigação. Piracicaba: SBEA/FUNEP.1, 63-120.

Silva, V. P. R., Campos, J. H. B. C., \& Azevedo, P. V. (2009). Wateruse efficiency and evapotranspiration of mango orchard grown in northeastern region of Brazil. Scientia Horticulturae, 1(120), 467-472.

Tagliaferre, C., Silva, J. P., Paula, A., Guimaraes, D. U. G., \& Barroso, N. I. S. (2012). Estimativa da evapotranspiração de referência para três localidades do Estado da Bahia. Revista Caatinga, 25(2), 136-143.

Turco, J. E. P., Faria, M. T., \& Fernandes, E. J. (2005). Influência da forma de obtenção do saldo de radiação na comparação de métodos de estimativa da evapotranspiração de referência. Revista Irriga, 10(3), 215-228.

Vescove, H. V., \& Turco, J. E. P. (2005). Comparação de Três Métodos de Estimativa da Evapotranspiração de Referência para a Região de Araraquara - SP. Irriga, 25(3), 713-721. 